\title{
Testing and Analysis of the Shear Modulus of Urea Granules
}

\author{
Jinfeng Wang ${ }^{1}$, Detang Zou ${ }^{2}$, Jinwu Wang ${ }^{1}$, and Wei Zhou ${ }^{1}$ \\ ${ }^{1}$ College of Engineering, Northeast Agricultural University, Harbin 150030, China \\ ${ }^{2}$ College of Agriculture, Northeast Agricultural University, Harbin 150030, China \\ \{ju_jinyan, zoudt, jinwuw\} a163.com, 592091975@qq.com
}

\begin{abstract}
In order to study the interaction between urea and mechanical structure, using direct shear test method, the shear modulus of urea was tested by C-LM4 computer measurement and control shear force measuring instrument. The obtained test curves show that: extrusion makes the binding strength between urea molecules reduce, shear force peak emerge, then decline rapidly; as rolling granules and surrounding granules formed compression and friction resistance in contact surface along the direction of shear, shear force emerges rise again. At the test temperature $22^{\circ} \mathrm{C}$, ambient humidity $45 \%$, the density of urea granules $1.33 \times 10^{3} \mathrm{~kg} / \mathrm{m}^{3}$, the values range of shear modulus of urea granules was measured, which was $0.23-0.3 \mathrm{GPa}$.
\end{abstract}

Keywords: urea, shear force, shear modulus, test.

\section{Introduction}

China is a big agricultural country, which is also a big fertilizer production and consumption country. Fertilizers play an important role in the development of agricultural production, especially in grain production. China uses less than $10 \%$ of the world's arable land to feed $22 \%$ of the world's population, which is inseparable with the contribution of fertilizer. For quite a long time, fertilizer is still an essential part to realizing agricultural sustainable development.

Rice is one of China's most important grain crops. Urea is widely applied in rice farming process, which is directly synthesized by raw materials of liquid ammonia and carbon dioxide under high temperature. The researches of urea are mainly concentrated in the application of urea in agriculture, the fertilizer proportion of urea and other fertilizers, the fertilizer utilization of urea, the synthesis of urea, and the chemical composition and so on, but the physical characteristics of urea are rarely researched[1-2]. Urea plays an important role in the process of rice farming, when applying fertilizer using paddy field fertilization machinery, urea often absorbs moisture, which results in urea has low flowability, adheres to fertilizer box and the conveying pipe, and leads to technical problems of fertilization rate reduced, fertilization quality decline, therefore, when researching the interaction of urea and mechanical structure, the physical characteristics of urea must be studied, such as 
density, elastic modulus, shear modulus and Poisson's ratio, etc. [3-5]. The shear modulus of urea was measured using direct shear test method, the shear modulus of urea was obtained, which provides related research methods and data reference for the research of physical characteristics of urea [6-9].

\section{Materials and Methods}

\subsection{Structure and Working Principle of Test Equipment}

Shear modulus test equipment includes shear force measuring instrument, data lines, measurement and control program, as shown in figure 1. The fixed shear-slice of the shear force measuring instrument was replaced by shear-slice of urea granules, shear force measuring instrument was connected with computer using dedicated data line, one end of data line was inserted into the computer USB port, the other end was connected to the rear 9-hole D-type data communication interface of the shear force measuring instrument. To open the power switch of shear force measuring instrument, and start the measuring and control program on the computer.

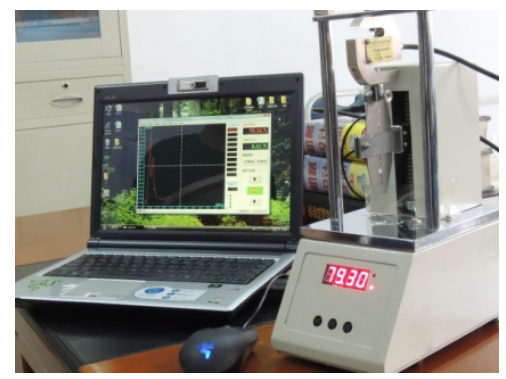

Fig. 1. Test equipment and test process of shear modulus

Click the "up" button of the measurement and control program of the computer, the activity shear frame of shear force measuring instrument was raised, which made the distance between the underneath of activity shear frame and the square hole of fixed shear-slice was more than $40 \mathrm{~mm}$. Urea granules, which will be measured, were fixed on shear-slice, click the "down" button, the instrument started to shear urea granules, when urea granules were shear off, the test was over. Measurement and control program of computer can record experiment data, and display curves.

\subsection{Experimental Equipment}

Shear modulus test equipment was mainly composed by shear force measuring instrument, data lines, measurement and control program.

Shear Force Measuring Instrument. C-LM4 computer measurement and control shear force measuring instrument was used, which was developed by northeast agricultural 
university, this instrument can measure seed, fertilizer and food from $0.05 \mathrm{~N}$ to $250 \mathrm{~N}$, the measurement accuracy was $< \pm 1 \%$, the shear speed was $5 \mathrm{~mm} / \mathrm{s}$, the voltage was $220 \mathrm{~V}$, frequency was $50 \mathrm{~Hz}$, the input power of electric machine was $20 \mathrm{~W}$.

Measurement and Control Program. Measurement and control program has operation control, test curve display, peak force maintain, test data management and other functions. The display window of test curve shows the time - shear force curve, which can display multiple samples test curve simultaneously, and has coordinate zoom function. Sampling rate of the measurement and control program is 100 test points / $\mathrm{s}$, sampling interval is 0.01 $\mathrm{s}$, and recorded data are stored into computer with Excel file format.

\subsection{Experimental Materials}

Experimental material is urea granules, which has bigger structure size. Circular urea fertilizer was grounded into cube by file and sandpaper, the length of cube was $2 \mathrm{~mm}$, $2.5 \mathrm{~mm}, 3 \mathrm{~mm}, 3.5 \mathrm{~mm}, 4 \mathrm{~mm}$ and $4.5 \mathrm{~mm}$, which were taken as test samples, the test temperature was $22^{\circ} \mathrm{C}$, ambient humidity was $45 \%$, the density of urea particle was 1.33 $\times 10^{3} \mathrm{~kg} / \mathrm{m}^{3}$.

\subsection{Experimental Methods}

Experimental method of shear modulus of urea was direct shear, the obtained data were the changing curves of the time and shear force by shear force measuring instrument, the line segment of linear elastic deformation was identified on the time - shear force curve [10-11], according to equation (1), shear modulus $G$ was obtained as follow:

$$
G=\frac{\tau}{\gamma}=\frac{\Delta Q / A}{v \cdot \Delta t / d}=\frac{d}{v A} \cdot \frac{\Delta Q}{\Delta t}=\frac{k d}{v A}
$$

Where $\Delta Q$ is the increment of shear force when urea granules linear elastic deformation, unit: $\mathrm{N} ; A$ is the area of shear surface of urea granules, unit: $\mathrm{mm}^{2} ; \Delta t$ is the increment of time when urea granules linear elastic deformation, unit: $\mathrm{s} ; \boldsymbol{v}$ is the moving velocity of activity shear frame, unit: $\mathrm{mm} / \mathrm{s} ; v \cdot \Delta t$ is the increment of displacement when urea granules linear elastic deformation, unit: $\mathrm{mm} ; d$ is the size of urea granules sample, unit: $\mathrm{mm} ; k$ is the ratio of the shear force increment and the time increment when urea granules linear elastic deformation, namely the slope of the linear elastic deformation on the time - shear force curve.

\section{$3 \quad$ Results and Analysis}

Different size of urea granules were selected to make shear test, the shear modulus of urea granules were measured, but due to the limited length of paper, only urea granules with side length $3.5 \mathrm{~mm}$ and $3 \mathrm{~mm}$ were analyzed in this paper. Three urea granules with side length $3.5 \mathrm{~mm}$ were selected to do shear test, the test curves were obtained by measurement and control program, as shown in figure 2. 


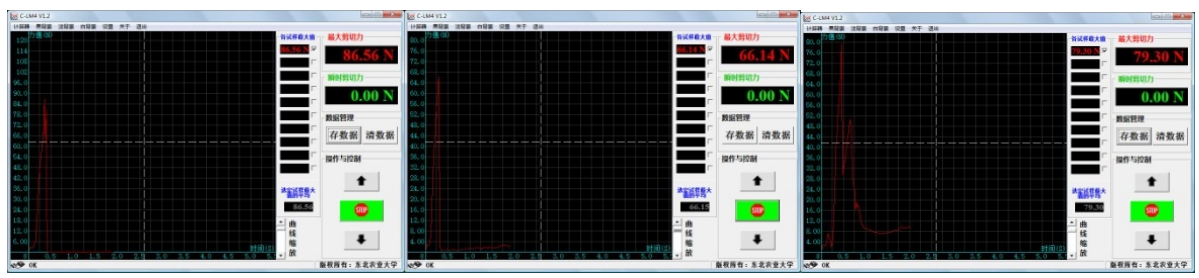

Fig. 2. The course of shear force - time of urea granules with side length $3.5 \mathrm{~mm}$

Three urea granules with side length $3 \mathrm{~mm}$ were selected to do shear test, the test curves were obtained by measurement and control program, as shown in figure 3 .

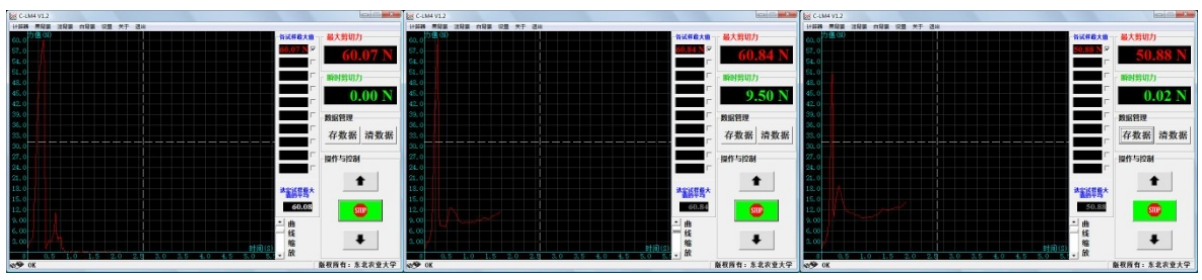

Fig. 3. The course of shear force - time of urea granules with side length $3 \mathrm{~mm}$

As shown in figure 2 and figures 3, With the moving of activity shear frame of shear force measuring instrument, firstly shear force increased rapidly, and reached a maximum, and then reduced rapidly, which had the same curve changing rule with the existing shear physical test. When activity shear frame contacted with urea granules, activity shear frame and fixed shear-slice surface had compression force to urea granules, but the action force between them was small, stress distribution of urea granules was uniform. With the moving of activity shear frame, sheared part formed shear zone with longitudinal width about $1 \mathrm{~mm}$, the extrusion force of activity shear frame to urea granules was enhanced, which made urea molecules in central part of the shear zone lost link, and appeared crack, shear force continued to increase, the peak of shear force appeared, and then decreased rapidly. With the increase of movement amount of activity shear frame, shear force of activity shear frame to urea granules further strengthened, shear zone was further squeezed, parts of granules, which lost connection force, rolled along the shear direction, and contacted with surrounding granules to form compression and friction resistance, shear force increased again. Finally, under the action of activity shear frame, the rest urea granules were further cracked to overcome frictional force between urea granules, which made urea granules between activity shear frames and fixed shear-slice staggered, shear force decreased to zero, urea granules were sheared off.

The obtained test data from the measurement and control program were fitted by Excel software, the curves of test points - shear force were obtained. The curve of test points- shear force of urea granules with side length $3.5 \mathrm{~mm}$ was obtained, as shown in figure 4 , The curve of test points - shear force of urea granules with side length $3 \mathrm{~mm}$ was obtained, as shown in figure 5. 


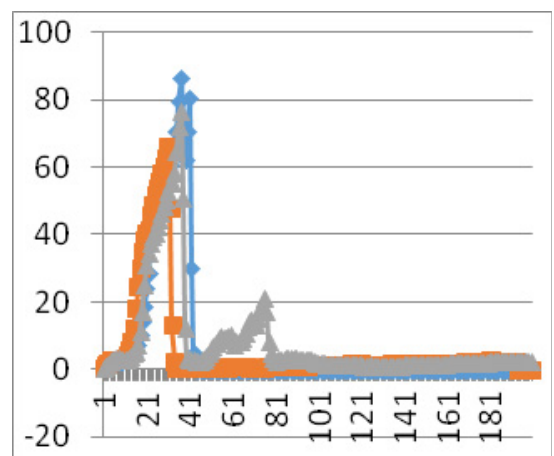

Fig. 4. Changing curves of test points - shear force of urea granules with side length $3.5 \mathrm{~mm}$

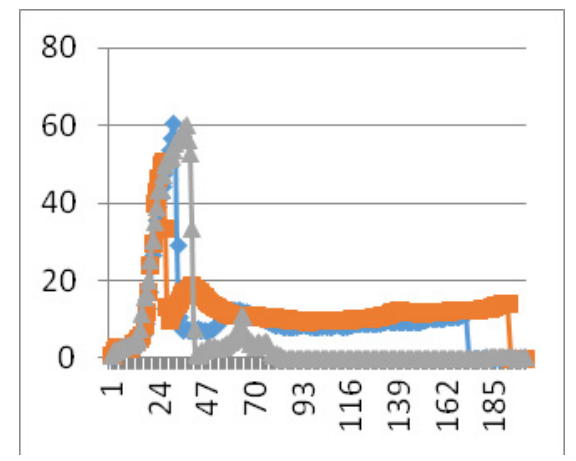

Fig. 5. Changing curves of test points - shear force of urea granules with side length $3 \mathrm{~mm}$

In figure 4 and figure 5, the horizontal axis indicated test points, there were 200 test points, the vertical axis indicated shear force. As the measurement and control program recorded a test point data every $0.01 \mathrm{~s}$, while the moving speed of activity shear frame of the shear force measuring instrument was $0.5 \mathrm{~mm} / \mathrm{s}$, so the horizontal axis can be converted into time, and also can be converted into displacement.

As shown in figure 5, the changing rule of the curve of test points - shear force was very similar to figure 4 , but different shear force peaks. In order to obtain the shear modulus of urea granules, a straight line should be identified on the test points - shear force curve before the first peak appeared. As shown in equation (1), the slope of this straight line can reflect the shear modulus of urea granules. Test points from 0 to 20 can't be selected as straight-line segment, because generally instrument exist error in the measurement range of $10 \%$ or urea granules specimen was not standard.

In order to find a segment of straight line on the test points - shear force curve, the experimental data of shear force, which present linear changing rule, were obtained from 6 groups test results of urea granules with side length of $3.5 \mathrm{~mm}$ and $3 \mathrm{~mm}$ by the measurement and control program, the test data were analyzed by Design-Expert6.0.1 software. 
Taking shear force as performance index, test time as factor, 25-30 data of test points of shear force were selected to fit curve, the function relationship between shear force and time was obtained, test data are shown in table 1.

Table 1. Test data of shear force

\begin{tabular}{|c|c|c|c|c|c|c|c|}
\hline \multirow{2}{*}{$\begin{array}{c}\text { test } \\
\text { points }\end{array}$} & \multirow{2}{*}{ time/s } & \multicolumn{6}{|c|}{ shear force/ } \\
\cline { 3 - 8 } & & 1 & 2 & 3 & 1 & 2 & 3 \\
\hline 25 & 0.115 & 40.2504 & 40.3289 & 39.4166 & 32.8009 & 29.9266 & 33.3914 \\
\hline 26 & 0.12 & 43.0561 & 41.6140 & 40.6919 & 33.5293 & 31.5293 & 36.3552 \\
\hline 27 & 0.125 & 46.1462 & 45.7735 & 42.5950 & 35.9144 & 35.8581 & 39.0242 \\
\hline 28 & 0.13 & 49.4620 & 49.1187 & 44.5570 & 39.1125 & 39.0168 & 41.4485 \\
\hline 29 & 0.135 & 51.8066 & 51.6889 & 46.4307 & 41.6435 & 39.3326 & 42.4581 \\
\hline 30 & 0.14 & 53.2094 & 54.0727 & 48.3339 & 44.4099 & 41.7269 & 44.2253 \\
\hline
\end{tabular}

The fitting regression curve of the influence of the test time to shear force was obtained for urea granules with side length $3.5 \mathrm{~mm}$ by Design-Expert 6.0 .1 software, as shown in figure 6.

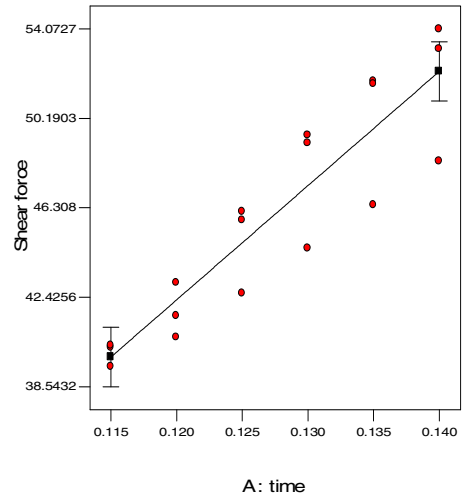

Fig. 6. The fitting regression curve of the influence of the test time to shear force for urea granules with side length $3.5 \mathrm{~mm}$

Variance analysis showed that model was significant, the time $t$ was a valid model items. The obtained regression equations is shown as follow:

$$
Q=496.03 t-17.21
$$

The slope of curve was 496.03 in equation (2), which was substituted into equation (1), the value of shear modulus $\mathrm{G}$ was obtained, which was $2.83 \times 10^{7} \mathrm{~Pa}$.

The fitting regression curve of the influence of the test time to shear force was obtained for urea granules with side length $3 \mathrm{~mm}$ by Design-Expert 6.0.1 software, as shown in figure 7. 


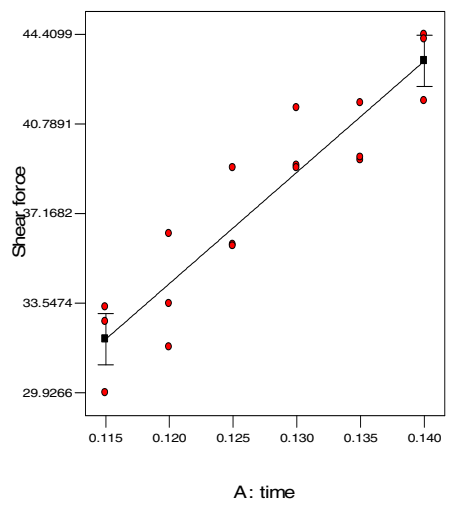

Fig. 7. The fitting regression curve of the influence of the test time to shear force for urea granules with side length $3 \mathrm{~mm}$

Variance analysis showed that model was significant, the time $\mathrm{t}$ was a valid model items. The obtained regression equations is shown as follow

$$
Q=450.09 t-19.67
$$

The slope of curve was 450.09 in equation (3), which was substituted into equation (1), the value of shear modulus $\mathrm{G}$ was obtained, which is $3 \times 10^{7} \mathrm{~Pa}$. Urea granules with side length $2-4.5 \mathrm{~mm}$ were selected to do experiment, the obtained values range of the shear modulus $\mathrm{G}$ is $2.3 \times 10^{7} \mathrm{~Pa}-3 \times 10^{7} \mathrm{~Pa}$.

\section{Conclusions}

(1) Urea granules was sheared off by C-LM4 computer measurement and control shear force measuring instrument, which was developed by northeast agricultural university, test data were obtained and test curves were displayed by measurement and control program of equipment. The obtained test curves show that: the compression between shear frame and urea granules makes the binding strength between urea molecules reduce, the peak of shear force emerge, then decline rapidly; as the rolling granules and surrounding granules formed compression and frictional shear force in contact surface along the shear direction, shear force emerges rise again.

(2) The obtained test data from the measurement and control program were fitted by Excel software, the test points - shear force curves were obtained. The regression equation of urea granules in the elastic deformation was fitted by Design-Expert software, the shear modulus of urea granules with side length $3.5 \mathrm{~mm}$ and $3 \mathrm{~mm}$ are respectively $2.83 \times 10^{7} \mathrm{~Pa}$ and $3 \times 10^{7} \mathrm{~Pa}$.

(3) At the test temperature $22^{\circ} \mathrm{C}$, ambient humidity $45 \%$, the density of urea particle $1.33 \times 10^{3} \mathrm{~kg} / \mathrm{m}^{3}$, the values range of shear modulus of urea granules was measured by shear force measuring instrument, which is from $2.3 \times 10^{7} \mathrm{~Pa}$ to $3 \times 10^{7} \mathrm{~Pa}$. 
Acknowledgment. This research was supported by National Natural Science Foundation of China (51205056), Youth Science Foundation of Heilongjiang Province (QC2011C007), Special Funds of Science and Technology Innovative Talents of Harbin (2012RFQXN004), Postdoctoral Fund Project of Heilongjiang Province (LBH-Z11222).

\section{References}

1. Li, Q., Jiang, X., Dai, H., et al.: Study on Properties of PVA Plasticized Urea /Formamide. China Plastics Industry 40(2), 24-26 (2012)

2. Cheng, Z., Yu, Z., Sun, X., et al.: Performance Analysis and Comparison for Radial with Axial Flow Urea Reactors. Chemical Fertilizer Design 49(6), 36-39 (2011)

3. Ding, J., Jin, X., Guo, Y., et al.: Study on 3-D Numerical Simulation for Soil Cutting with Large Deformation. Transactions of the Chinese Society for Agricultural Machinery 38(4), 118-121 (2007)

4. Ding, Y., Zhang, X., Wang, S.: Preparation and Properties of Porous Bioceramics Made of Bovine Bone. China Ceramics 45(11), 28-31 (2009)

5. Yu, H.: Synthesis and Properties of Carbon Microtubes. Harbin Institute of Technology (2011)

6. Blunden, B.G., McLachlan, C.B., Kirby, J.M.: A High-Speed Shear Box Machine. Journal of Agricultural Engineering Research 56(1), 81-87 (1993)

7. Wheeler, P.N., Godwin, R.J.: Soil Dynamics of Single and Multiple Tines at Speeds Up to 20 km/h. Journal of Agricultural Engineering Research 63(3), 243-250 (1996)

8. Onwualu, A.P., Watts, K.C.: Draught and Vertical Forces Obtained from Dynamic Soil Cutting by Plane Tillage Tools. Soil \& Illage Research 48(4), 239-253 (1998)

9. Mootaz, A.-E., Hamilton, R., et al.: 3D Dynamic Analysis of Soil-Tool Interaction Using the Finite Element Method. Journal of Terramechanics 40(1), 51-62 (2003)

10. Karmakar, S., Ashrafizadeh, S.R., Kushwaha, R.L.: Experimental Validation of Computational Fluid Dynamics Modeling for Narrow Tillage Tool Draft. Journal of Terramechanics 46(5), 277-283 (2009)

11. Yang, W., Cai, G., Yang, J.: Dynamics Simulation of Direct Shear Test. Transactions of the Chinese Society for Agricultural Machinery 42(7), 96-101 (2011) 